\title{
Role of lymph node dissection in the management of upper tract urothelial carcinomas: a meta-analysis
}

\author{
Runqi Guo ${ }^{\dagger} \mathbb{D}$, Yuze Zhu ${ }^{\dagger}$, Gengyan Xiong, Xuesong Li, Kai Zhang ${ }^{*}$ and Liqun Zhou*
}

\begin{abstract}
Background: Lymph node dissection (LND) is not routinely performed during radical nephroureterectomy (RNU) in upper tract urothelial carcinomas (UTUC) and the role of LND has been controversial. We aim to investigate whether patients with LND had improved survival in UTUC patients.

Methods: We performed a systematic literature search of PubMed, Embase, and Cochrane library for citations published prior to January 2016, describing LND performed among UTUC patients and conducted a standard meta-analysis of survival outcomes.

Results: Eleven eligible studies containing 7516 patients satisfied the inclusion criteria. Pooled HRs for cancerspecific survival (CSS) and recurrence-free survival (RFS) were $1.17(P=0.18)$ and $1.33(P=0.19)$ respectively. However, the patients in the LND group had more advanced tumour stages and grades $(P<0.001)$. Further subgroup analysis showed that among muscle-invasive UTUC patients, the pooled HR for CSS and RFS were 1.10 $(P=0.42)$ and $0.92(P=0.72)$ respectively. Besides, no difference was found in CSS and RFS between pNO and pNx individuals in overall populations and in patients with muscle-invasive UTUC, while pN+ patients had significantly worse prognosis when compared to pNO patients.
\end{abstract}

Conclusions: LND during RNU allows more accurate staging and prediction of survival, but it remains uncertain whether LND independently improves survival in patients with UTUC. However, standard use of LND should be further investigated in a multi-center, prospective evaluation to obtain a definitive statement regarding this matter.

Keywords: Lymph node dissection, Recurrence, Survival, Upper urinary tract, Urothelial carcinoma

\section{Background}

Urothelial carcinomas are the fourth most common tumors [1]. However, upper tract urothelial carcinomas (UTUC) are comparatively uncommon compared to bladder cancer and occupy only $5-10 \%$ of urothelial carcinomas [2, 3]. Approximately $30 \%$ of patients suffered from muscle-invasive UTUC at presentation and the incidence of lymph node metastasis ranges from 30\% to $40 \%$ at surgery $[4,5]$.

Radical nephroureterectomy (RNU) with bladder cuff resection and regional lymph node dissection (LND) is the

\footnotetext{
* Correspondence: kaizhangpku@163.com; zhoulqmail@sina.com ${ }^{\dagger}$ Equal contributors

Department of Urology, Peking University First Hospital and Institute of Urology, National Research Center for Genitourinary Oncology, Beijing 100034, China
}

backbone of management $[3,6,7]$. Generalizing results from previous bladder cancer researches [8-13], it seems reasonable to believe that LND in conjunction with RNU may provide not only utile staging and prognostic information but also a therapeutic benefit in selected patients with UTUC. Nevertheless, the therapeutic benefit of LND in improving survival remains controversial [14-16].

For these reasons, we systematically reviewed the published studies and performed a meta-analysis of studies in which data were reported for the treatment of LND to assess whether patients who achieved LND had improved cancer-specific survival (CSS) or recurrencefree survival (RFS) compared with patients who did not achieve LND, as a means for providing data for standardizing the indication of LND and assisting in creating a better management strategy for UTUC. 


\section{Methods}

\section{Search strategy}

We systematically reviewed PubMed, Embase, and Cochrane library for citations published prior to January 2016, describing LND performed among patients with UTUC. The search strategy included the terms: lymphadenectomy or lymph node excision or lymphatic metastases, and upper tract urothelial neoplasms or upper tract urothelial cancer or transitional cell carcinoma of the upper urinary tract. Two authors independently reviewed article titles and abstracts for eligibility, and divergences were settled by consensus.

\section{Inclusion and exclusion criteria of trials}

Studies were included if they met all the following criteria: (1) prospective randomized studies or welldesigned non-randomized controlled experiments; (2) studies analyzing the relationship between LND and UTUC prognosis; (3) clearly described outcome assessment by representing it in CSS or RFS; (4) sufficient survival information with hazard ratios (HR) and corresponding 95\% confidence interval (CI), or Kaplan-Meier curves comparing survival among pathologic subgroups that were stratified according to LN status ( $\mathrm{pN} 0$, negative node; $\mathrm{pNx}$, skipping LND; $\mathrm{pN}+$, positive node) or between LND and NLND; and (5) demographics and pathologic characteristics of patients were stratified according to LN status or according to the presence or absence of LND. Studies were excluded if they met one of the following criteria: (1) the article was a review or meta-analysis; (2) No available data could be able to extracted from the previously published studies; (3) the article deal with recurrent UTUC, metastatic carcinoma, previous or concurrent invasive bladder tumors or neoadjuvant chemotherapy; and (4) (potentially) overlapping study populations were reported for the same outcome.

\section{Data extraction}

All studies identified were independently reviewed by two reviewers. Titles and abstracts were screened for initial inclusion and final agreement on study inclusion was made by discussion and consensus with other authors. Two reviewers extracted data from all the included studies independently. Divergences were settled through consensus.

\section{Quality assessment}

The quality of the cohort studies was evaluated using the modified Newcastle-Ottawa Scale, which met the demands of this study [17]. This scale assesses risk in three domains: patient selection, comparability of LND and NLND groups and assessment of outcome (Table 1). To compare the two cohorts, we
Table 1 Newcastle-Ottawa quality assessment scale

Check list
Selection
•How representative was the control group (lymph node dissection)
in comparison with the general elderly population for transitional cell
carcinoma of the upper urinary tract? (if yes, one point; no point, if
the patients were selected or selection of group was not described)
•How representative was the research group (non-lymph node
dissection) in comparison with the elderly population for transitional
cell carcinoma of the upper urinary tract? (if data from the same
community as the control group, one point; no point, if drawn from
a different source or selection of group was not described)
•Assignment for treatment: any detail report? (if yes, one point)
Comparability
•Group comparable for the grade of tumor, clinical TNM staging
system (if yes, two points; one point was assigned, if one of these
two characteristics had differences; no star was assigned, if the two
groups differed)
•Group comparable for age, gender (if yes, two points; one star was
assigned, if one of these two characteristics had differences; no
point was assigned, if the two groups differed)
- Comprehensively evaluated the outcome? (yes, one point for
of Diseases; no point, if this information was not reported)
-

concentrated on the following variables that had been identified as independent predictors in previous multivariate studies: age, gender, tumor grade and tumor stage [18-21]. Each study was assessed by two reviewers independently. Any divergences were settled by discussion or through arbitrament by a third reviewer if no agreement could be reached.

\section{Data analysis and synthesis}

We use log HR and the variance as the summary outcome measure from all trials in the meta-analysis. For each trial, HR with the $95 \%$ CI of the survival rate was derived and calculated using either the fixedeffects model or the random-effects model [22]. Chisquare test was used to assess the heterogeneity between studies. For $P$ values less than 0.1 , the assumption of homogeneity was deemed invalid. Therefor, we calculated pooled estimates using random effects modeling, which provides more conservative estimates than fixed effects modeling when heterogeneity was present.

Publication bias is considered as a concern for metaanalyses. In our study, publication bias was assessed by funnel plots and Egger's regression [23]. Review manager version 5.3 (Cochrane Collaboration, Oxford, UK) was used for data analysis. A $P$ value of less than 0.05 was considered statistically significant. 


\section{Results}

\section{Study identification and quality assessment}

A total of 658 studies were identified. After excluding duplicates, 144 articles remained, 127 of which were excluded: 106 were apparent irrelevant studies, 4 were case, series/case reports, and 17 were letters/reviews/ comments. 17 were reviewed in depth, and a full examination of the text was performed. Five studies were excluded because of insufficient outcome and one was excluded due to potentially overlapping study populations. At last, 11 studies involving 7516 UTUC patients were included into this meta-analysis $[14,15,16,24-30$, 31] (Fig. 1) (Table 2).

The quality assessment of included cohort studies was performed using the modified Newcastle-Ottawa Scale. Studies that scored $>7$ were considered as having low risk of bias, scores of 5-7 indicated moderate risk of bias, and scores of $<5$ indicated high risk of bias, and the total scores are shown in Table 3. Most studies were deemed to be of moderate risk of bias and we only scored 3 of 11 studies as having low risk of bias. Commonly identified concern was the comparability of LND and NLND groups, especially regarding tumor grade and TNM staging.

\section{Meta-analysis results}

\section{Cancer-specific survival}

Of the 10 studies that referred to CSS, there was significant heterogeneity among them $\left(I^{2}=80 \%, C h i^{2}=45.96\right.$, $P<0.00001)$. Thus, a random-effects model was used to calculate the pooled HR and corresponding 95\% CI. No

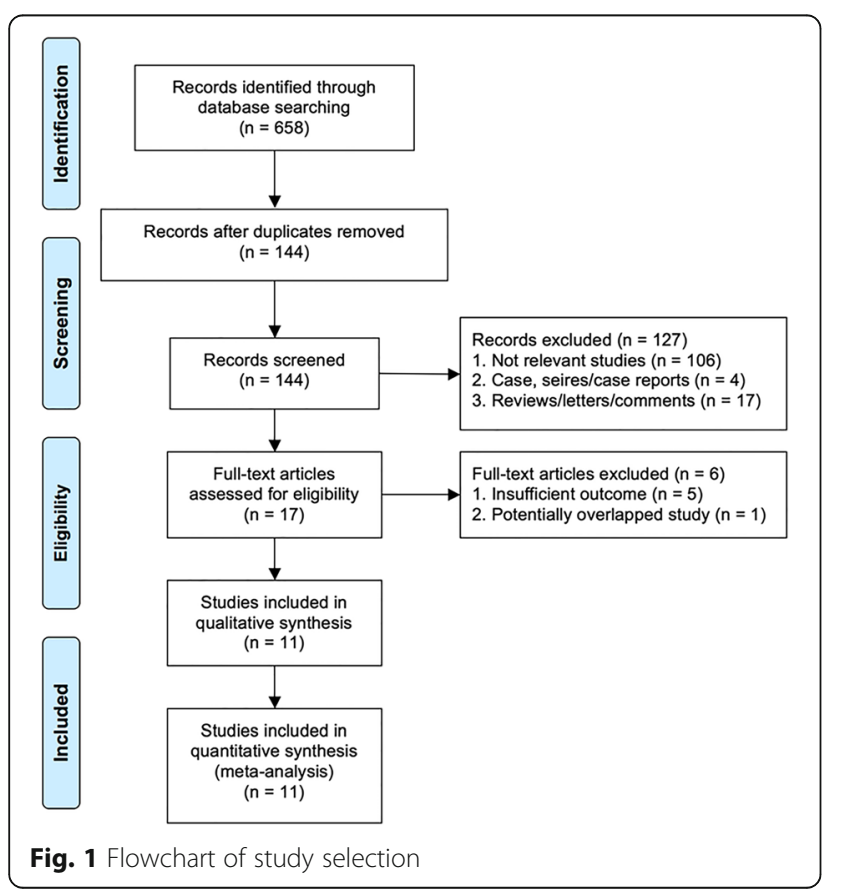

statistically significance was found between the LND group and the NLND group $(\mathrm{HR}=1.17,95 \% \mathrm{CI}$ : $0.93-$ $1.48, P=0.18$ ) (Fig. 2 A1). Besides, patients with pN0 did not have better CSS compared with those with $\mathrm{pNx}$ (HR $=0.99, \quad 95 \%$ CI: 0.81-1.22, $P=0.95)$ with significant heterogeneity $\left(I^{2}=94 \%, C h i^{2}=35.97, P<0.00001\right)$ (Fig. 2 A2), while patients with $\mathrm{pN}+$ showed poor CSS compared with those with pN0 $(\mathrm{HR}=3.38,95 \% \mathrm{CI}$ : 1.94-5.89, $P<0.0001)$ with significant heterogeneity $\left(I^{2}=93 \%, C h i^{2}=71.90, P<0.00001\right)$ (Fig. 2 A3).

To explore the source of apparent heterogeneity, we compared the differences in tumor stage and tumor grade between the groups, thereby demonstrating the features between the groups using Chi-square tests and Fisher's exact tests for categorical variables (Table 4). The results showed that there was remarkable significant difference in tumor stage and tumor grade between the LND group and NLND group $(P<0.001)$, which might have significant association with the heterogeneity.

\section{Recurrence-free survival}

Significant heterogeneity was observed in the four studies that focused on RFS $\left(I^{2}=89 \%, C h i^{2}=26.57, P\right.$ $<0.00001)$, hence we utilzed the random-effects model. The pooled HR for RFS was 1.33 (95\% CI: 0.87-2.06, $P=0.19$ ), which indicate that LND was not associated was better RFS in patients with UTUC (Fig. 2 B1). Meanwhile, in consideration of $\mathrm{pN} 0 / \mathrm{pNx}$, no significant difference in RFS between $\mathrm{pNO}$ and $\mathrm{pNx}$ was found $(\mathrm{HR}=0.98,95 \% \mathrm{CI}: 0.62-1.55, P=$ $0.93)$ and there was significant heterogeneity among them $\left(I^{2}=85 \%, C h i^{2}=19.82, P=0.0002\right)$ (Fig. 2 B2). In contrast, $\mathrm{pN}+$ showed poor RFS compared with those pN0 ( $\mathrm{HR}=3.46,95 \% \mathrm{CI}: 2.00-5.97, P<0.0001)$ with significant heterogeneity $\left(I^{2}=78 \%, C h i^{2}=13.95\right.$, $P<0.003$ ) (Fig. 2 B3).

\section{Subgroup analysis}

We performed subgroup analysis according to pT statuses, among patients with muscle-invasive UTUC. Data for CSS in patients with muscle-invasive UTUC were reported in four studies, and there was heterogeneity among those studies $\left(I^{2}=63 \%, C h i^{2}=8.04, P=0.05\right)$; hence, we utilzed the random-effects model. However, no statistically significance was found between the two groups $(\mathrm{HR}=$ 1.10, 95\% CI: 0.88-1.37, $P=0.42$ ) (Fig. 2 C1).

Additionally, the results of the subsequent analyses showed no difference in RFS between the LND group and the NLND group among muscle-invasive UTUC individuals $(\mathrm{HR}=0.92,95 \% \mathrm{CI}: 0.58-1.46, P=0.72)$ and there was relatively high heterogeneity in this subgroup $\left(I^{2}=88 \%, C h i^{2}=16.48, P=0.0003\right)$ (Fig. $2 \mathrm{C} 2$ ).

Furthermore, among the patients with muscleinvasive UTUC, no significant difference between pN0 


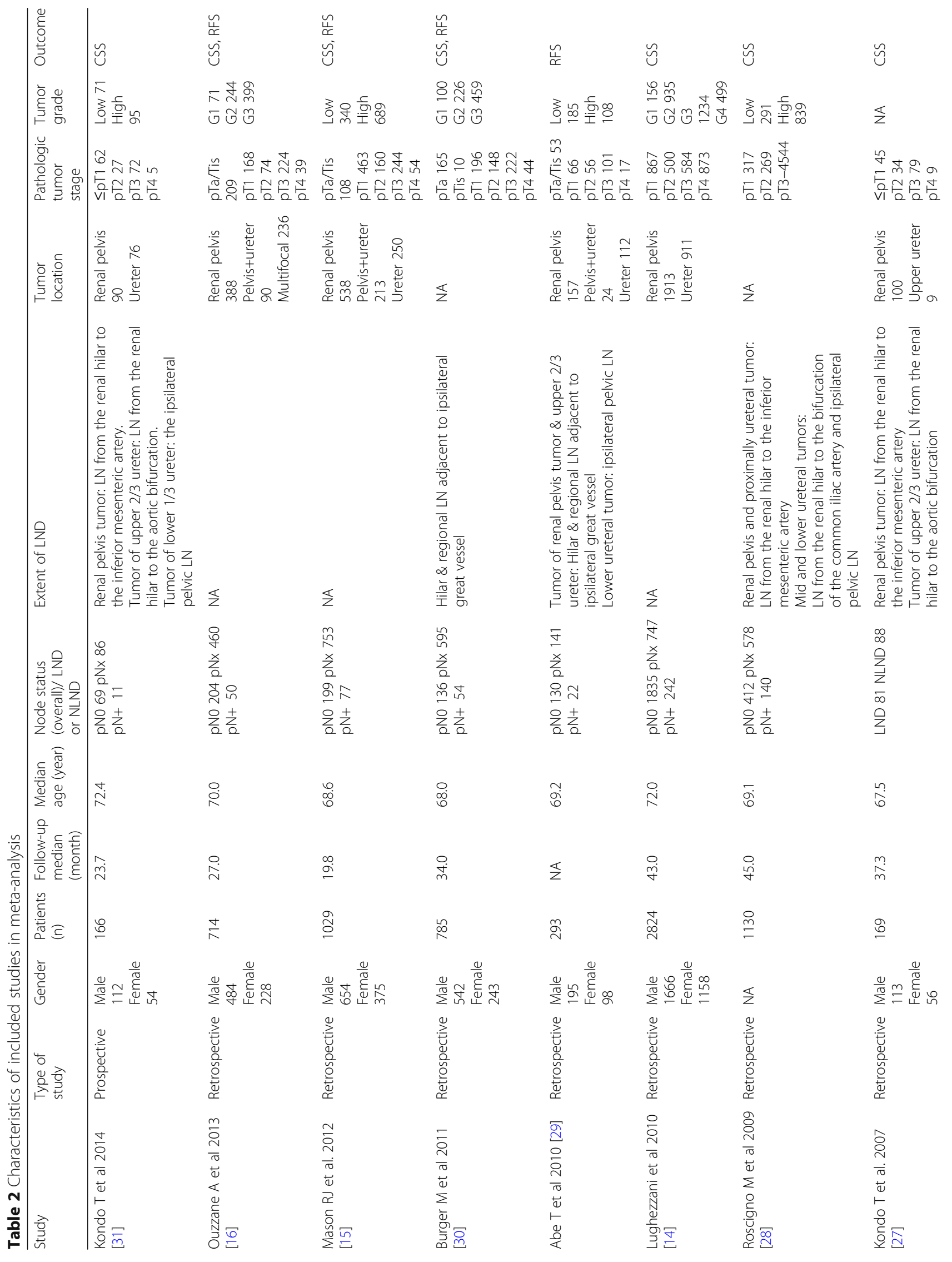




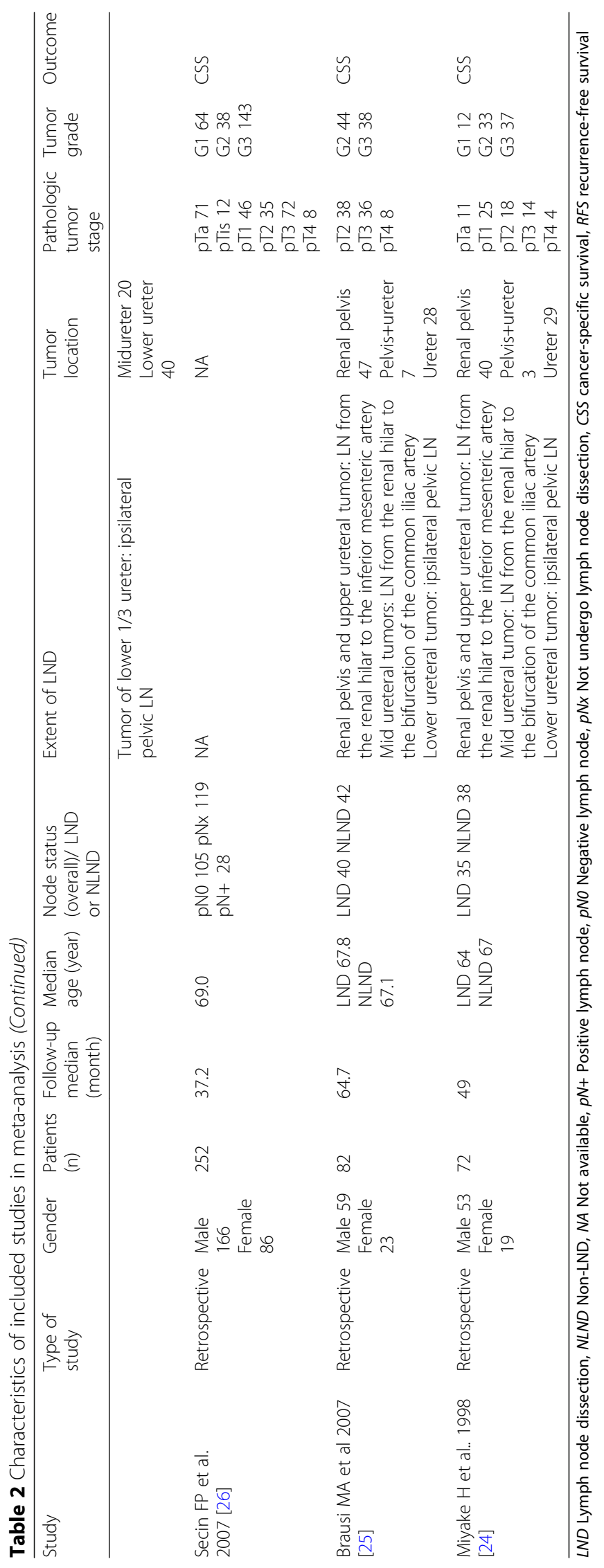


Table 3 Assessment for quality of included studies

\begin{tabular}{|c|c|c|c|c|c|c|c|c|}
\hline \multirow[t]{2}{*}{ Study } & \multicolumn{3}{|c|}{ Selection } & \multicolumn{2}{|c|}{ Comparability } & \multicolumn{2}{|c|}{ Outcome assessment } & \multirow[t]{2}{*}{ Score } \\
\hline & 1 & 2 & 3 & 1 & 2 & 1 & 2 & \\
\hline Kondo T et al 2014 [31] & 1 & 1 & 1 & 2 & 0 & 1 & 1 & 7 \\
\hline Ouzzane A et al 2013 [16] & 1 & 1 & 1 & 0 & 2 & 1 & 1 & 7 \\
\hline Mason RJ et al 2012 [15] & 1 & 1 & 1 & 0 & 1 & 1 & 1 & 6 \\
\hline Burger M et al 2011 [30] & 1 & 1 & 1 & 0 & 1 & 1 & 1 & 6 \\
\hline Abe T et al 2010 [29] & 1 & 1 & 1 & 0 & 2 & 1 & 1 & 7 \\
\hline Lughezzani et al 2010 [14] & 1 & 1 & 1 & 0 & 2 & 1 & 1 & 7 \\
\hline Roscigno M et al 2009 [28] & 1 & 1 & 1 & 0 & 1 & 1 & 1 & 6 \\
\hline Kondo T et al 2007 [27] & 1 & 1 & 1 & 1 & 2 & 1 & 1 & 8 \\
\hline Secin FP et al 2007 [26] & 1 & 1 & 1 & 1 & 1 & 1 & 1 & 7 \\
\hline Brausi MA et al 2007 [25] & 0 & 1 & 1 & 2 & 2 & 1 & 1 & 8 \\
\hline Miyake H et al 1998 [24] & 1 & 1 & 1 & 2 & 1 & 1 & 1 & 8 \\
\hline
\end{tabular}

and $\mathrm{pNx}$ was found in CSS and RFS ( $\mathrm{HR}=0.97,95 \%$ CI: $0.64-1.47, P=0.87$; and $\mathrm{HR}=0.97$, 95\% CI: $0.64-$ $1.47, P=0.87$, respectively) and there was significant heterogeneity $\left(I^{2}=94 \%, C h i^{2}=35.97, P<0.00001\right.$; and $I^{2}=89 \%, C h i^{2}=18.80, P<0.00001$, respectively) (Fig. 2 $\mathrm{C} 3$ \& 2C4). However, patients with $\mathrm{pN}+$ showed poor CSS and RFS in comparison with those with pNO (HR = 3.27, 95\% CI: $2.83-3.78, P<0.00001$; and $\mathrm{HR}=2.10$, 95\% CI: 1.05-4.20, $P=0.0002$, respectively) (Fig. 2 C5and C6).

\section{Publication bias}

The publication bias was detected using a funnel plot of the meta-analysis result. The basic symmetry of the funnel plots suggested that there was no obvious publication bias (Fig. 3). The Egger's test for CSS and RFS did not show any evidence of publication bias.

\section{Discussion}

Radical cystectomy with pelvic LND for muscle-invasive bladder cancer is relatively standardized because it improves tumor staging and survival of patients [32, 33]. However, potential benefit of LND during RNU on survival for UTUC is still controversial $[15,30]$. On the basis of the latest European guidelines on UTUC, LND should be performed in conjunction with RNU not only for better tumor staging but also for prognosis improvement [3]. Nevertheless, this recommendation is only Level III evidence. Thus, we reviewed the published studies and conducted a meta-analysis to clarify the prognostic value of LND in patients with UTUC.

In the present research, 11 studies were eligible and the HRs of cumulative survival rates were summarized quantitatively. Our analysis revealed that $\mathrm{pN}+$ patients had significantly worse prognosis when compared to pNO patients. The same results were observed when restricting the analyses to patients with muscle-invasive carcinomas, who should, anyway, be systematically considered for staging LND in light of this growing body of data.

However, no difference was found in survival or disease recurrence when comparing $\mathrm{pN} / \mathrm{pNx}$ individuals and the LND/NLND groups. The sample size of the included studies could explain these results. Most of the early years studies include small numbers of patients (less than 200), while larger series (more than 1000) with more events only emerged recently. Besides, the decision to perform LND was left to the discretion of the surgeon, it is possible that those NLND patients had less aggressive disease than LND patients, and that a true benefit to LND does exist. An increased risk of cancerrelated death is usually related to higher tumor stage and grade. In this comparison, there was no significant difference in CSS and RFS between the LND group and the NLND group, which may reversely suggest the possible therapeutic value of LND for patients with more aggressive tumors. Nevertheless, the results remained no significant difference when controlling for tumor stage. Conversely, in a review by Kondo and Tanabe [34], it was highlighted that when the regional nodes were completely dissected, the patients with the advanced stage had significantly higher survival compared with those without LND.

Interestingly, $\mathrm{pNx}$ was not associated with poor CSS and RFS in patients with muscle-invasive carcinomas and in overall population. Several explanations may account for our results. First, pNx individuals were most likely identified by their surgeons as low risk for nodal metastases. It is also possible that $\mathrm{pNx}$ individuals may harbor micrometastatic lymph node deposits, which could be either destroyed or removed during the surgery, without being identified as pN1 by the pathologist. Furthermore, the lack of standardized anatomical limits 


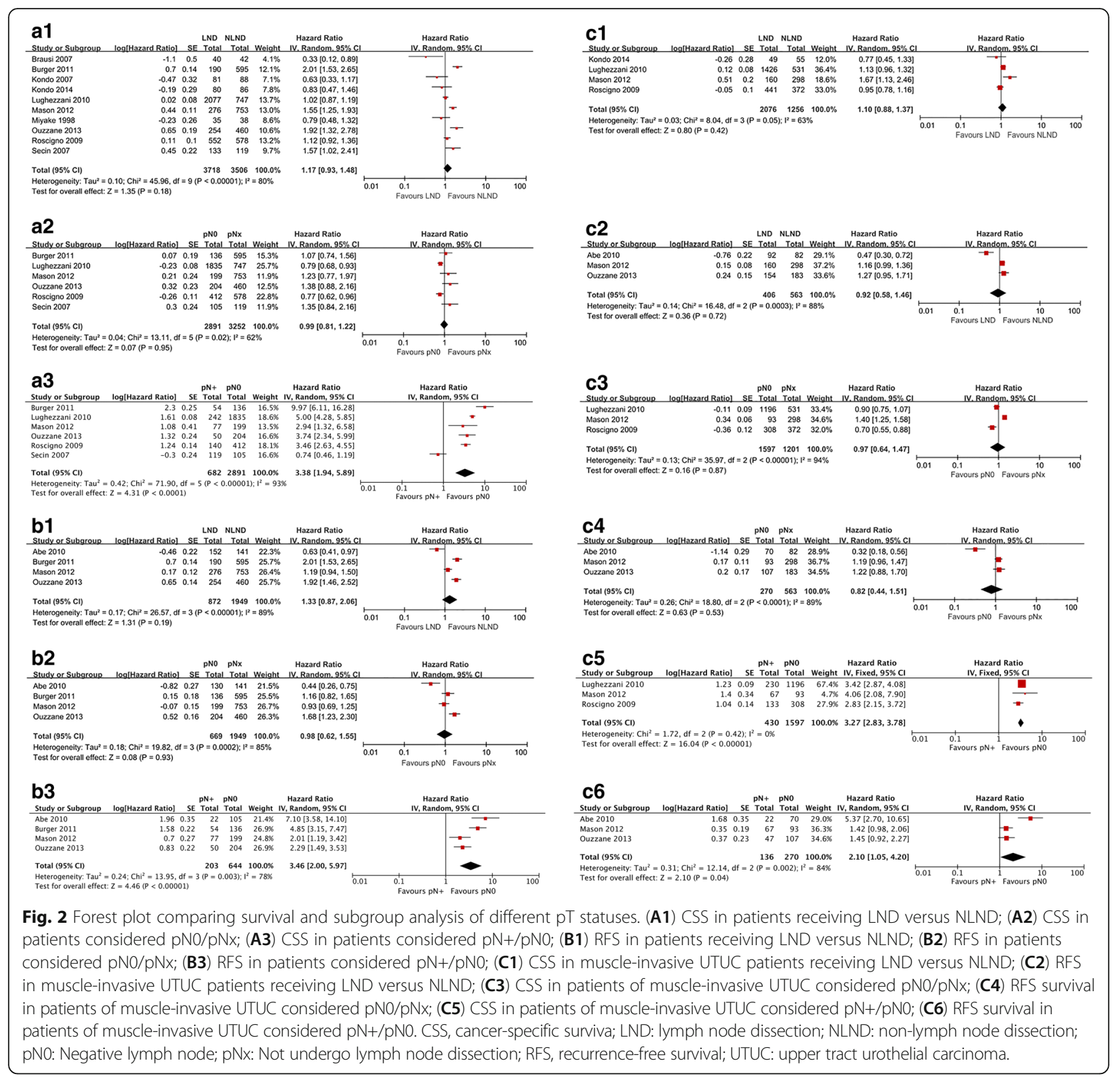

Table 4 Chi-square tests for two groups

\begin{tabular}{llll}
\hline Variable & $\operatorname{LND}(\mathrm{n}, \%)$ & $\operatorname{NLND}(\mathrm{n}, \%)$ & $P$ value \\
\hline Tumor stage & & & $<0.001$ \\
$\leq \mathrm{T} 1$ & $1210(31.3)$ & $1684(46.2)$ & \\
T2 & $722(18.7)$ & $637(17.5)$ & \\
T3 & $1204(31.2)$ & $990(27.2)$ & \\
T4 & $726(18.8)$ & $335(9.2)$ & \\
Tumor grade & & & \\
Low grade or $\leq \mathrm{G} 2$ & $1610(35.4)$ & $1281(43.2)$ & \\
High grade or $>$ G2 & $2936(64.6)$ & $1682(56.8)$ & \\
\hline
\end{tabular}

LND lymph node dissection, NLND non LND and indication for the LND could account for our results: some patients certainly had very limited dissection and unsuitable for tumor location, leading to a wrong histological report of pNO stage even though they had nodal metastasis not including in the LND.

It is noteworthy that $49.0 \% \mathrm{RNU}$ patients were staged as pNx in our studies. In 2009, Roscigno et al. pointed out that patients with pN0 disease had a better prognosis than $\mathrm{pNx}$ disease in patients with muscle-invasive carcinomas [28]. It is conceivable that, despite a higher $\mathrm{pNx}$ rate at tertiary care centers, the extent of LND in those in whom it was performed was substantially greater than the LND extent in the community. Under this premise, a more important stage migration towards 


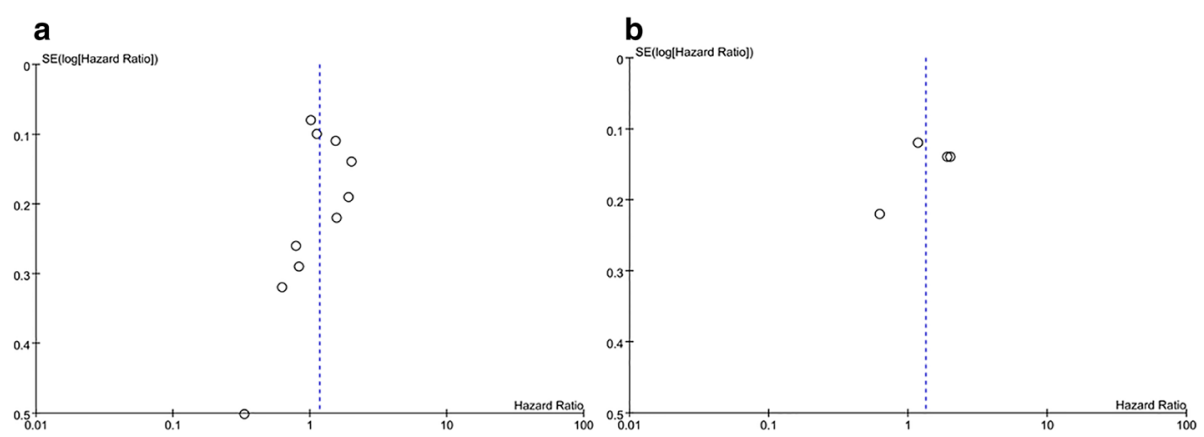

Fig. 3 Funnel plot for the evaluation of potential publication bias. (a) cancer-specific survival; (b) recurrence-free survival

true pN0 status may have occurred at tertiary care centers than in the current population-based series [14]. Taken together, our findings suggest that $\mathrm{pNx}$ individuals have no better prognosis than pN0 individuals.

Without standardized criteria for who should receive LND and how extensive LND should be, comparisons between series proved to be challenging. It was reported that the patients with incomplete LND in showed lower survival than those with complete LND, which reached statistical significance. Five-year CSS in the patients with pT2 or higher and pT3 or higher was $77.9 \%$ and $73.2 \%$ in the patients with complete LND, but just $54.0 \%$ and $43.7 \%$ in those with incomplete LND and $59.0 \%$ and $47.3 \%$ in those with NLND [34].

The most important finding of our study is that LND patients had no worse prognosis than NLND patients, especially in those with muscle-invasive carcinomas. According to a recent review, carrying out LND for UTUC is unlikely to be time-consuming and to increase the risk of major complications [34]. Although the current quality of evidence is generally not high, which may lead to biased and uncertain results, it might still suggest that the role of LND in UTUC is of importance, as UTUC is likely to simulate the biological behavior of bladder cancer because of the same histology among the two diseases.

Limitation should also be considered. First, the sources of the publications were limited, thus potentially introducing inevitable publication bias. Second, although 11 eligible studies involving 7516 patients were included in this meta-analysis, most of them were retrospective studies and the sample sizes of some selected studies are small, which might render the results less reliable. Third, marked heterogeneity of studies was seen in pooled-analysis of CSS and RFS. Furthermore, 7 of the 11 included studies provided the extent of LND, but the indication and extent of LND were not standardized. Last but not the least, as the included studies spanned a 10-year interval, the year in which the surgery occurred could be associated with different survival rates due to better imaging, earlier diagnosis and improved perioperative strategies of care. In the future, the role of LND should be further examined by validating templates of regional lymph nodes, and by prospective studies with larger numbers of patients. Then, we will discuss whether LND can be a standard treatment for UTUC.

\section{Conclusions}

LND during RNU allows more accurate staging and prediction of survival, but it remains uncertain whether LND independently improves survival in patients with UTUC. However, standard use of LND should be further investigated in a multi-center, prospective evaluation to obtain a definitive statement regarding this matter.

\section{Abbreviations}

Cl: Confidence interval; CSS: Cancer-specific survival; HR: Hazard ratios; LND: Lymph node dissection; RFS: Recurrence-free survival; RNU: Radical nephroureterectomy; UTUC: Upper tract urothelial carcinomas

\section{Acknowledgements}

This work was supported by grants from the Collaborative Research Foundation of Peking University Health Science Center and National Taiwan University, College of Medicine (BMU20120318), Natural Science Foundation of Beijing (7152146) and the Clinical Features Research of Capital (No.Z151100004015173).

Ethic approval and consent to participate

The study was not primary research involving humans or animals but was a secondary analysis of human subject data available in the public domain.

\section{Funding}

Not applicable.

\section{Availability of data and materials}

Not applicable.

\section{Authors' contributions}

GRQ participated in data collection and management, data analysis and manuscript writing. ZYZ participated in data analysis and manuscript writing. XGY participated in data collection and manuscript writing. LXS participated in project development, critical revision and manuscript editing. ZK participated in project development, critical revision and supervision. ZLQ participated in project development, critical revision and supervision. All the authors approved the final manuscript. 


\section{Competing interest}

The authors declare that they have no competing interests.

\section{Consent for publication}

Not applicable.

\section{Publisher's Note}

Springer Nature remains neutral with regard to jurisdictional claims in published maps and institutional affiliations.

Received: 11 October 2016 Accepted: 13 March 2018 Published online: 10 April 2018

\section{References}

1. Oxford Centre for Evidence-based Medicine Levels of Evidence (May 2009). Produced by bob Phillips, Chris ball, Dave Sackett, Doug Badenoch, Sharon Straus, Brian Haynes, Martin Dawes since November 1998. Updated by Jeremy Howick March 2009.

2. Siegel RL, Miller KD, Jemal A. Cancer statistics, 2015. CA Cancer J Clin. 2015; 65:5-29.

3. Rouprêt M, Babjuk M, Compérat E, Zigeuner R, Sylvester RJ, Burger $M$, et al European Association of Urology guidelines on upper urinary tract urothelial cell carcinoma: 2015 update. Eur Urol. 2015;68:868-79.

4. Corrado F, Ferri C, Mannini D, Corrado G, Bertoni F, Bacchini P, et al. Transitional cell carcinoma of the upper urinary tract: evaluation of prognostic factors by histopathology and flow cytometric analysis. J Urol. 1991;145:1159-63.

5. Schatteman $P$, Chatzopoulos C, Assenmacher C, De Visscher L, Jorion JL, Blaze $V$, et al. Laparoscopic nephroureterectomy for upper urinary tract transitional cell carcinoma: results of a Belgian retrospective multicentre survey. Eur Urol. 2007:51:1633-8.

6. NCCN Clinical practice guidelines in oncology: bladder cancer. Including upper tract tumors and urothelial carcinoma of the prostate. Version 2.2015. National Comprehensive Cancer Network Web site http://www.ncen.org/ professionals/physician gls/pdf/bladder.pdf. Accessed May 2015.

7. Cornu JN, Rouprêt M, Carpentier X, Geavlete B, de Medina SG, Cussenot O, et al. Oncologic control obtained after exclusive fexible ureteroscopic management of upper urinary tract urothelial cell carcinoma. World J Urol. 2010;28:151-6.

8. Koppie TM, Vickers AJ, Vora K, Dalbagni G, Bochner BH. Standardization of pelvic lymphadenectomy performed at radical cystectomy: can we establish a minimum number of lymph nodes that should be removed? Cancer. 2006;107:2368-74

9. Leissner J, Hohenfellner R, Thüroff JW, Wolf HK. Lymphadenectomy in patients with transitional cell carcinoma of the urinary bladder: significance for staging and prognosis. BJU Int. 2000:85:817-23.

10. Herr HW, Bochner BH, Dalbagni G, Donat SM, Reuter VE, Bajorin DF. Impact of the number of lymph nodes retrieved on outcome in patients with muscle invasive bladder cancer. J Urol. 2002;167:1295-8.

11. Konety BR, Joslyn SA, O'Donnell MA. Extent of pelvic lymphadenectomy and its impact on outcome in patients diagnosed with bladder cancer: analysis of data from the surveillance, epidemiology and end results program data base. J Urol. 2003:169:946-50.

12. Abdel-Latif M, Abol-Enein H, El-Baz M, Ghoneim MA. Nodal involvement in bladder cancer cases treated with radical cystectomy: incidence and prognosis. J Urol. 2004;172:85-9.

13. Liedberg F, Mansson W. Lymph node metastasis in bladder cancer. Eur Urol. 2006:49:13-21

14. Lughezzani $G$, Jeldres $C$, Isbarn $H$, Shariat SF, Sun M, Pharand D, et al. A critical appraisal of the values of lymph node dissection at nephroureterectomy for upper tract urothelial carcinoma. Urology. 2010;75:118-24.

15. Mason RJ, Kassouf W, Bell DG, Lacombe L, Kapoor A, Jacobsen N, et al. The contemporary role of lymph node dissection during nephroureterectomy in the management of upper urinary tract urothelial carcinoma: the Canadian experience. Urology. 2012;79:840-5.

16. Ouzzane A, Colin P, Ghoneim TP, Zerbib M, De La Taille A, Audenet F, et al. The impact of lymph node status and features on oncological outcomes in urothelial carcinoma of the upper urinary tract (UTUC) treated by nephroureterectomy. World J Urol. 2013;31:189-97.
17. Taggart DP, D'Amico R, Altman DG. Effect of arterial revascularisation on survival: a systematic review of studies comparing bilateral and single internal mammary arteries. Lancet. 2001;358:870-5.

18. Clements T, Messer JC, Terrell JD, Herman MP, Ng CK, Scherr DS, et al. Highgrade ureteroscopic biopsy is associated with advanced pathology of upper-tract urothelial carcinoma tumors at definitive surgical resection. J Endourol. 2012;26:398-402.

19. Li CC, Chang TH, Wu WJ, Ke HL, Huang SP, Tsai PC, et al. Significant predictive factors for prognosis of primary upper urinary tract cancer after radical nephroureterectomy in Taiwanese patients. Eur Urol. 2008;54:1127-34.

20. Lughezzani G, Burger M, Margulis V, Matin SF, Novara G, Roupret M, et al. Prognostic factors in upper urinary tract urothelial carcinomas: a comprehensive review of the current literature. Eur Urol. 2012;62:100-14.

21. Shariat SF, Godoy G, Lotan Y, Droller M, Karakiewicz PI, Raman JD, et al. Advanced patient age is associated with inferior cancer-specific survival after radical nephroureterectomy. BJU Int. 2010;105:1672-7.

22. DerSimonian R, Laird N. Meta-analysis in clinical trials. Control Clin Trials. 1986;7:177-88.

23. Green S. Cochrane handbook for systematic reviews of interventions version 5.1.0 [updated march 2011]. The Cochrane collaboration. Chichester: Wiley; 2011.

24. Miyake H, Hara I, Gohji K, Arakawa S, Kamidono S. The significance of lymphadenectomy in transitional cell carcinoma of the upper urinary tract. Br J Urol. 1998:82:494-8.

25. Brausi MA, Gavioli M, De Luca G, Verrini G, Peracchia G, Simonini G, et al. Retroperitoneal lymph node dissection (RPLD) in conjunction with nephroureterectomy in the treatment of infiltrative transitional cell carcinoma (TCC) of the upper urinary tract: impact on survival. Eur Urol. 2007:52:1414-8.

26. Secin FP, Koppie TM, Salamanca Jl, Bokhari S, Raj GV, Olgac S, et al. Evaluation of regional lymph node dissection in patients with upper urinary tract urothelial cancer. Int J Urol. 2007;14:26-32.

27. Kondo T, Nakazawa H, Ito F, Hashimoto Y, Toma H, Tanabe K. Impact of the extent of regional lymphadenectomy on the survival of patients with urothelial carcinoma of the upper urinary tract. J Urol. 2007:178:1212-7.

28. Roscigno M, Shariat SF, Margulis V, Karakiewicz P, Remzi M, Kikuchi E, et al. Impact of lymph node dissection on cancer specific survival in patients with upper tract urothelial carcinoma treated with radical nephroureterectomy. J Urol. 2009;181:2482-9.

29. Abe T, Shinohara N, Muranaka M, Sazawa A, Maruyama S, Osawa T, et al. Role of lymph node dissection in the treatment of urothelial carcinoma of the upper urinary tract: multi-institutional relapse analysis and immunohistochemical re-evaluation of negative lymph nodes. Eur J Surg Oncol. 2010:36:1085-91.

30. Burger M, Shariat SF, Fritsche HM, Martinez-Salamanca Jl, Matsumoto K, Chromecki TF, et al. No overt influence of lymphadenectomy on cancerspecific survival in organ-confined versus locally advanced upper urinary tract urothelial carcinoma undergoing radical nephroureterectomy: a retrospective international, multi-institutional study. World J Urol. 2011;29:465-72.

31 Kondo T, Hara I, Takagi T, Kodama Y, Hashimoto Y, Kobayashi H, et al. Template-based lymphadenectomy in urothelial carcinoma of the renal pelvis: a prospective study. Int J Urol. 2014;21:453-9.

32 Dhar NB, Klein EA, Reuther AM, Thalmann GN, Madersbacher S, Studer UE. Outcome after radical cystectomy with limited or extended pelvic lymph node dissection. J Urol. 2008;179:873-8.

33 Holmer M, Bendahl PO, Davidsson T, Gudjonsson S, Månsson W, Liedberg F. Extended lymph node dissection in patients with urothelial cell carcinoma of the bladder: can it make a difference? World J Urol. 2009;27:521-6.

34 Kondo T, Tanabe K. Role of lymphadenectomy in the management of urothelial carcinoma of the bladder and the upper urinary tract. Int J Urol. 2012:19:710-21. 